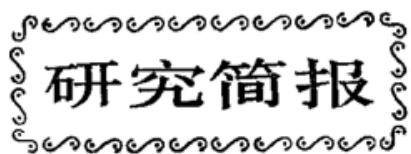

\title{
内点方法的线性规划分解原则和算法 $(\mathbf{I})^{*}$
}

\author{
魏 紫 銮 \\ (中国科学院计算中心, 北京 100080)
}

\section{关键词 内点方法、线性规划、分解原则、分解算法}

\section{1 引 言}

出现在资源分配、生产计划、土地、森林管理以及经济和军事等方面的线性规划模型中,它 们的共同之点都是由许多个独立的大的复杂的子系统满足一整体的约束组成的系统. 已有许 多求解这类特殊结构问题的方法. Dantzig-Wolfe 分解原则所导出的分解算法是解决这一问 题的较为有效的方法 ${ }^{[1-3]}$, 它是利凸多面体中的任一点可以表示为其顶点的凸组合和极射线的 非负组合的性质, 把原问题转换为求解一系列规模较小的线性规划问题. 并通过原始问题与 其对偶问题之间关系的信息, 求得问题的最优解。它的主要缺点是由算法产生的点列在最优 解的邻域内收玫得太慢以及数值计算的不稳定性. 近年来各种不同形式的求解线性规划问题: 的内点方法已被广为研究 ${ }^{[4]}$, 许多数值试验的结果表明对许多大规模的线性规划问题, 内点法 比单纯形法更有吸引力. 由于内点法是从可行区域的内部搜索至最优点, 从而避免了单纯形 法沿着区域边界游访众多顶点而达到极值点的过迴路径. 而由严格可行解至最优解的向量可 以表示为许多线性无关的可行方向的线性组合. 因而与上述的分解算法相似，原来的问题可 以转换为计算各独立子系统的可行方向和求解一系列其相应的规模较小的规划问题.

本文提出内点方法意义下的线性规划问题的分解原则以及在仿射均衡尺度变换所构造的: 分解算法,同时证明了算法的收敛性.

\section{2 内点方法下的分解原则}

本节推导在可行区域内点非空假设下的线性规划的一般分解原则. 不失一般性, 考虑以 下的线性规划问题.

LP:

$$
\begin{aligned}
& \min z=c \cdot x, \\
& \text { s. t. }\left\{\begin{array}{l}
A x \leqslant b, \\
B x=b_{1}, \\
x \geqslant 0,
\end{array}\right.
\end{aligned}
$$

其中 $A$ 和 $B$ 分别是 $m \times n, m_{1} \times n$ 的矩阵, $b \in R^{m}$ 和 $b_{1} \in R^{m_{1}}$ 分别是给定 向 量. $x \in R^{*}$, 
$a \cdot b$ 表示任意向量 $a, b$ 的内积. 为了方便, 假设集合 $\Omega_{1}=\{x: A x \leqslant b, x \geqslant 0\}$ 和 $Q_{2}=$ $\left\{x: B x-b_{1}, x \geqslant 0\right\}$ 是非空的, 且 $Q_{1}^{0}, Q_{2}^{0}$ 分别表 $\Omega_{1}, Q_{2}$ 的非空严格内点集合, 目标函数在 $Q_{1} \cap \boldsymbol{Q}_{2}$ 上是有界的.

假设存在一内点 $\bar{x} \in Q_{i}^{0} \cap Q_{2}^{n}$, 并以 $L$ 表示 $B$ 的笭化空间. 我们希望找到一向量 $u \in L$ 使 得 $x=\bar{x}+u \geqslant 0$, 且其相应的目标函数值是下降的, 即有 $z(x)<z(\bar{x})$. 如果以上式替代 $L P$ 中的向量 $x$, 则原问题就变为以 $u$ 为变量的以下 $L P_{1}$ 问题:

$\mathrm{LP}_{1}$ :

$$
\begin{aligned}
& \min z(u)=z(\ddot{x})+c \cdot u, \\
& \text { s. t. }\left\{\begin{array}{l}
A u \leqslant \bar{b}, \\
B u=0, \\
u \geqslant-\bar{x},
\end{array}\right.
\end{aligned}
$$

其中 $z(\bar{x})-c \cdot \bar{x}, \bar{b}=b-A \bar{x}>0$, 显然 $\mathrm{LP}_{1}$ 是等价于 LP. 假设 $u^{*}$ 是 $\mathrm{LP}_{1}$ 的一最优 解, $y_{0}, y_{1}, y_{2}$ 分别是约束(1), (2)和(3)式相应的拉格朗日乘子向量,则由最优性条件可见以下 关系式成立.

$$
\left\{\begin{array}{l}
A^{T} y_{0}+B^{T} y_{1}+y_{2}=c, \\
y_{0} \cdot\left(\bar{b}-A u^{*}\right)=0, \\
y_{2} \cdot\left(\bar{x}+u^{*}\right)=0, \\
y_{0} \leqslant 0, y_{2} \geqslant 0 .
\end{array}\right.
$$

反之,如果存在向量 $y_{0} \leqslant 0$ 和 $u^{*} \in L$, 使得 $\bar{x}+u^{*} \geqslant 0$ 和(5)式成立,则考虑以下线性规划:

$\mathrm{LP}_{2}$ :

$$
\min u v=\left(\bar{x}+u^{*}\right) \cdot y_{2} \text {, s.t, } B^{T} y_{1}+y_{2}-c-A^{T} y_{0}, y_{2} \geqslant 0 .
$$

如果对给定的 $y_{0} \leqslant 0, u^{*} \in L$, 问题 $\mathrm{LP}_{2}$ 的极小值为零, 则 (4)-(7)式成立. 另一方面, $\mathrm{LP}_{2}$ 的对偶问题可以表示为以下的 LD:

$$
\max u w=\left(c-A^{T} y_{0}\right) \cdot u \text {, s.t. } B u-0, u u \leqslant \hat{x},
$$

其中 $1=\bar{x}+u^{*}$. 显然, 当 $\mathrm{LP}_{2}$ 的极小值为零时, $\mathrm{LD}$ 的极大值亦为零. 由此有以下结论.

定理 1 假设问题 LP 是可行的, 其目标函数值在 $\Omega_{1} \cap \Omega_{2}$ 上是有界的, 且 $\Omega_{1}^{0} \cap \Omega_{2}^{0} \neq$ ф. 假定存在一 $\bar{x} \in \Omega_{1}^{0} \cap Q_{2}^{0}$ 和 $u \in L, y_{0}$ 是约束(1)式相应的拉格朗日乘子向量. 则 $\hat{x}=\bar{x}+u$ 是 $L P$ 的最优解当且仅当 LD 的极大值为零.

定理的证明可由以上的叙述直接得到. 其结论可以推广到其它特殊结构形式的线 性 规 划.

\section{3 分 解 算 法}

根据分解原则本节提出仿射均衡尺度变换下的分解算法. 我们考虑以下的线性规划问题

$$
\text { (P): } \min u z=\sum_{i=1}^{l} c_{i} \cdot x_{i} \text {, s.t. } \sum_{i=1}^{l} A_{i} x_{i}=b, x_{i} \in Q_{i}, i \in \bar{L},
$$

其中 $\Omega_{i}=\left\{x_{i}: B_{i} x_{i}=b_{i}, x_{i} \geqslant 0, x_{i} \in R^{n_{i}}\right\}, A_{i} \in R^{m \times m_{i}}, B_{i} \in R^{m_{i} \times n_{i}}, c_{i} \in R^{n_{i}}, b_{i} \in R^{m_{i}}$, $i \in \bar{L}=\{1,2, \cdots, i\}, b \in R^{m}$ 分别是给定的. $x \in R^{n}=R^{n_{1}} \times R^{n_{2}} \times \cdots \times R^{n_{l}}$ 是末知向 量. 令 $\bar{Q}=\Omega_{1} \times \Omega_{2} \times \cdots \times \Omega_{l}, \Omega_{0}=\left\{x: \sum_{i=1}^{l} A_{i} x_{i}=b, x \geqslant 0, x \in R^{*}\right\}, \Omega-\Omega_{0} \cap \bar{\Omega}$. 假 设问题 $(P)$ 满足以下条件: (i) $Q$ 非空有界,且 $Q^{0}=\{x: x>0, x \in Q\}$ 非空, 


$$
\bar{m}=m+\sum_{i=1}^{l} m_{i}<n, u m<i .
$$

(ii) 矩阵 $B_{i} D_{i}$ 对所有 $x_{i} \in Q_{i}(i \in \bar{L})$ 满秩, 矩阵 $\left(A_{1} D_{1}, A_{2} D_{2}, \cdots, A_{l} D_{l}\right)$ 对 $x \in Q$ 满秩, 其 中 $\theta_{i}-\operatorname{diag}\left(x_{i}\right)$. (iii) 向量 $c_{i}-A_{i}^{T} y_{0}-B_{i}^{T} y_{i}(i \in \bar{L})$ 对 $y \in R^{\bar{m}}$ 至多有 $\bar{m}$ 个零分量, $y^{T}=\left(y_{0}^{T}, \cdots y_{l}^{T}\right)$.

今假定有初始严格内点 $\bar{x} \in Q^{0}$, 如果 $\bar{x}+h \in \Omega$, 称 $h$ 是 $\bar{x}$ 点的可行方向. 类似信赖域的 方法可以构造以 $\bar{x}$ 为中心的椭球 $S=\left\{x: \sum_{i=1}^{l}\left\|D_{i}^{-1}\left(x_{i}-\bar{x}_{i}\right)\right\|_{2}^{2} \leqslant \beta^{2}<1, x \in Q\right\}$, 在 $S$ 上极小 化目标函数就得到另一内点, 依此类推, 就可以产生一收玫于最优解的点列. 现在我们考虑如 何确定可行方向 $h$,由 $S$ 的定义可见

$$
x_{i}=\bar{x}_{i}+h_{i}=\bar{x}_{i}+D_{i} u_{i \bullet}
$$

应用仿射均衡尺度变换, 极小化 $S$ 上的目标函数的问题可以表示为 $\left(\boldsymbol{P}^{\prime}\right)$ :

$$
\begin{aligned}
& \min z(u)=z(\bar{x})+\sum_{i=1}^{l} c_{i} D_{i} u_{i}, \\
& \text { s. t. }\left\{\begin{array}{l}
\sum_{i=1}^{l} A_{i} D_{i} u_{i}=0, \\
B_{i} D_{i} u_{i}=0, i \in \bar{L}, \\
\beta^{2}-u \cdot u \geqslant 0,
\end{array}\right.
\end{aligned}
$$

其中 $z(\vec{x})=\sum_{i=1}^{l} c_{i} \cdot \bar{x}_{i}, u^{T}=\left(u_{1}^{T}, \cdots, u_{l}^{T}\right)$. 由最优性条件 $u^{*}$ 是 $\left(P^{\prime}\right)$ 的最优解当且仅当 存在 $\left(y^{*}\right)^{T}=\left(y_{0}^{* T}, \cdots, y_{l}^{* T}\right)$ 使以下条件

成立. 由(10)和(12)式可见

$$
\begin{gathered}
D_{i} A_{i}^{T} y_{0}^{*}+D_{i} B_{i}^{T} y_{i}^{*}-\sigma u_{i}^{*}=D_{i} c_{i}, i \in \bar{L}, \\
\sigma\left(\beta^{2}-u \cdot u\right)=0, \sigma \geqslant 0
\end{gathered}
$$

由以上关系式可以推得当 $\sigma \neq 0$ 时

$$
y_{i}^{*}=\left(B_{i} D_{i}^{2} B_{i}^{T}\right)^{-1} B_{i} D_{i}^{2}\left(c_{i}-A_{i}^{T} y_{0}^{*}\right) .
$$

$$
u_{i}^{*}=-\beta \frac{P_{i} D_{i}\left(c_{i}-A_{i}^{T} y_{0}^{*}\right)}{\delta_{N}}=-\beta \bar{u}_{i}^{*},
$$

其中 $P_{i}=I-D_{i} B_{i}^{T}\left(B_{i} D_{i}^{2} B_{i}^{T}\right)^{-1} B_{i} D_{i}, \quad \delta_{N}=\left(\sum_{i=1}^{l}\left\|P_{i} D_{i}\left(c_{i}-A_{i}^{T} y_{0}^{*}\right)\right\|_{2}^{2}\right)^{1 / 2}$, 而且 $\sigma=\delta_{N} / \beta$. 如果 $\sigma=0$, 则 $u_{i}^{*}=\lambda_{i} \bar{u}_{i}^{*}, \lambda_{i} \in[0, \beta)$. 由此可见如果 $y_{0}^{*}$ 是已知, 则所有 $u_{i}^{*}$ 均可确定. 为 计算 $y_{i}^{*}$ 之值定义以下函数

$$
F\left(y_{0}\right)=\sum_{i=1}^{l}\left\|P_{i} D_{i}\left(c_{i}-A_{i}^{T} y_{0}\right)\right\|_{2}^{2}
$$

显然, $F\left(y_{0}\right)$ 是 $R^{m}$ 上的一凸函数, 因为 $D_{i} P_{i} D_{i}(i \in \bar{L})$ 是正定或正半定的矩阵, 故存在一极 小解 $y_{0}^{*}$ 使

$$
y_{0}^{*}-\operatorname{Arg} \min _{y_{0} \in R^{m}} F\left(y_{0}\right) .
$$

综上所叙, 求解问题 $(P)$ 的分解算法简述如下: 假设存在初始点 $x^{(0)} \in Q^{0}$ (见文献 [5]), 
$\boldsymbol{y}_{0}^{(0)} \in R^{m}$ 和正数 $\beta<1$ 是给定的, 则算法的主要循环叙述如下:

算法 A（第 $k$ 次迭代）

(i) 定义

$$
D_{i}^{(k)}=\operatorname{diag}\left(x_{i, 1}^{(k)}, x_{i, 2}^{(k)}, \cdots, x_{i, n_{i}}^{(k)}\right), i \in \vec{L},
$$

计算投影矩阵 $P(k), i \in \bar{L}$.

(ii) 产生方向向量

$$
\bar{u}_{i}^{(k)}=u_{i}^{(k)} /\left\|u^{(k)}\right\|_{2}
$$

其中 $u_{i}^{(k)}=P_{i}^{(k)} D_{i}^{(k)}\left(c_{i}-A_{i}^{T} y_{0}^{(k)}\right),\left\|u^{(k)}\right\|_{2}-\left(\sum_{i=1}^{t} u_{i}^{(k)} \cdot u_{i}^{(k)}\right)^{1 / 2}$. 如果 $\left\|u^{(k)}\right\|_{2}=0$, 或 $\Delta z_{k-1}=$ 0 ,则 $x^{(k)}$ 是问题 $(P)$ 的一最优解,终止; 否则执行下一步.

(iii) 计算函数 $F_{k}\left(y_{0}\right)$ 的极小解 $\bar{y}_{0}^{(k)}$, 以 $\bar{y}_{0}^{(k)}$ 取代 $y_{0}^{(k)}$ 按照 (ii) 中的公式计算方向 向量 $\bar{u}_{i}^{(k)}$. 转步骤 (iv).

(iv) 求解以下的线性规划问题 $(\bar{P})$ :

$$
\begin{aligned}
& \min \Delta z_{k}=-\sum_{i=1}^{l} \bar{c}_{i}^{(k)} \lambda_{i}^{(k)}, \\
& \text { s. t. }\left\{\begin{array}{l}
-\sum_{i=1}^{l} \bar{a}_{i}^{(k)} \lambda_{i}^{(k)}=0, \\
\bar{\beta}^{2}-\left(\alpha_{i}^{(k)} \lambda_{i}^{(k)}\right)^{2} \geqslant 0, i \in \bar{L},
\end{array}\right.
\end{aligned}
$$

其中 $u_{i}^{(k)}=\lambda_{i}^{(k)} \bar{u}_{i}^{(k)}, \bar{c}_{i}^{(k)}=c_{i} D_{i}^{(k)} \bar{u}_{i}^{(k)}, \bar{a}_{i}^{(k)}-A_{i} D_{i}^{(k)} \bar{u}_{i}^{(k)}, \bar{\beta}<\beta . \quad \alpha_{i}^{(k)}=\bar{u}_{i}^{(k)} \cdot \bar{u}_{i}^{(k)} \geqslant 0$, 若 $\alpha_{i}^{(k)}=0$, 令 $\lambda_{i}^{(k)}=0$.

设 $\bar{\lambda}^{(k)}$ 是 $(\bar{P})$ 的最优解, $\hat{y}_{0}^{(k)}$ 是其相应的拉格朗日乘子向量. 令 $y_{0}^{(k+1)}=\hat{y}_{0}^{(k)}$.

(v) 生成新的内点

$$
x_{i}^{(k+1)}=x_{i}^{(k)}-\lambda_{i}^{(k)} D_{i}^{(k)} \bar{u}_{i}^{(k)} .
$$

由上述可见算法 $\mathrm{A}$ 可以分为二大步, 即计算 $\bar{y}_{0}^{(k)}, u^{(k)}$ 和极小化问题 $(\bar{P})$.

\section{参考文 献}

[ 1] 马仲謷、权龄、赖炎连,数学规划讲义,中国人民大学出版社, 1981 .

[2] Dantzig, G. B., Wolfe, P., Operations Res., 1960, 8:101-111.

[3] Ho, J. K., Math. Prog. Study, 1987, 31:119-128.

[4] Megiddo, N. et al., Progress in Mathematical Programming: Interior-point and Related MethodsSpringer-Verlag, New York Inc., 1989.

[5] Wei, Z. L., J. of Computational Mathematics, 1990, 8:16-22. 